NOUVELLE

\section{Quand les microbes se mêlent de la maladie de Parkinson}

\author{
Yanis Khenniche ${ }^{1}$, Esther Lutete ${ }^{2}$, Pierre Bobé ${ }^{3}$
}

${ }^{1} \mathrm{Ml}$ Biologie-Santé et magistère de biologie d’Orsay, Université Paris-Saclay, 91405 Orsay, France;

${ }^{2}$ Ml Biologie-Santé, Université Paris-Saclay 91405 Orsay, France ;

${ }^{3} \mathrm{ICPH}$ (interactions cellulaires et physiopathologie hépatique), Inserm UMR S1174, Université Paris-Saclay, bâtiments 440-443, rue des Adèles, 91400 Orsay, France.

yanis.khenniche@u-psud.fr

esther.lutete@u-psud.fr

pierre.bobe@u-psud.fr

\section{Le microbiote intestinal ${ }^{l}$}

Les systèmes physiologiques de l'homme sont souvent décrits séparément d'un point de vue fonctionnel et anatomique bien qu'ils interagissent de façon permanente, que ce soit lors du développement ou à l'âge adulte. Le système digestif, par exemple, interagit étroitement avec le microbiote intestinal ${ }^{2}$ (MI) pour son développement. Ces microorganismes, en symbiose avec l'organisme, communiquent avec les différents systèmes de l'hôte [1]. Ils sont très variables d'un individu à l'autre. De manière surprenante, ces bactéries sont reconnues et tolérées par le système immunitaire qui les distingue des bactéries pathogènes ${ }^{3}$.

\section{Interactions entre le système nerveux} central et le système digestif

Le développement du système nerveux est influencé par le $\mathrm{MI}$ et le système digestif: des facteurs neurotrophiques modulent la physiologie intestinale et, réciproquement, la sécrétion de neuropeptides par le système digestif a un impact sur le cerveau [2]. L'intestin grêle est la première source de dopamine et de sérotonine, deux neuromodulateurs très importants du système nerveux central (SNC) [3]. Le MI régule

Cette Nouvelle fait partie d'une série de 12 Nouvelles rédigées par les étudiants du Master «Biologie Santé » de I'université Paris-Saclay, qui sont parues dans les numéros 6-7, 8-9, 10 et 11 (2017) de médecine/sciences.

${ }^{1}$ Voir à ce propos le numéro thématique de médecine/ sciences consacré à ce thème en novembre 2016.

${ }^{2}$ Microbiote intestinal: ensemble des populations de microorganismes présents principalement dans le côlon, mais aussi l'intestin grêle.

3 Pathogène : élément étranger pouvant provoquer une pathologie et qui entraine une réaction immunitaire. aussi les systèmes de neurotransmission du SNC en modulant l'expression des récepteurs de la sérotonine et des récepteurs du glutamate de type NMDA (acide Nméthyl-D-aspartique) impliqués dans la plasticité synaptique [4]. $\varepsilon$ n outre, il participe activement au développement du SNC, et notamment à celui de l'hippocampe [5].

Altération de l'axe cérébro-intestinal : dysbiose et maladie de Parkinson L'étude de systèmes comme le SNC ou encore l'intestin - dans des conditions physiologiques ou lors de dysfonctionnements - doit donc prendre en compte leurs interactions réciproques. Celles-ci pourraient intervenir dans la genèse de troubles anxieux, dépressifs, mais aussi de troubles du spectre autistique $[6,7]$. Les recherches sur l'axe cérébro-intestinal sont donc pertinentes dans le cadre de l'exploration des maladies neurologiques. Par exemple, une étude révèle que le MI est altéré chez des patients atteints de maladie de Parkinson, suggérant que la dysbiose ${ }^{4}$ du MI pourrait être impliquée dans le développement de cette maladie [8].

\section{La maladie de Parkinson}

Aujourd'hui, il n'existe pas de traitement curatif de la maladie de Parkinson. Bien qu'il existe des formes génétiques ( $5 \%$ ), cette maladie se développe le plus souvent de manière sporadique ( $95 \%$ ). Elle se caractérise par une dégénérescence spécifique des neurones dopaminergiques

\footnotetext{
${ }^{4}$ Dysbiose (intestinale) : perturbation en termes de quantité et de qualité de la population microbienne du MI.
}

de la substance noire. Ces neurones sont responsables de la coordination de la réponse motrice en régulant notamment le cortex moteur et le cervelet. Une atteinte de cette structure entraîne des déficits moteurs prononcés: tremblements, rigidité, difficulté à déclencher les mouvements, lenteur d'exécution, défaut de coordination, etc. La dégénérescence des neurones dopaminergiques est provoquée par l'agrégation d'une protéine appelée $\alpha$-synucléine ( $\alpha$ Syn). Ces structures insolubles (corps de Lewy) provoquent la destruction des neurones et l'activation des cellules microgliales ${ }^{5}$, à l'origine d'une neuro-inflammation caractéristique de la maladie de Parkinson. Celle-ci est protectrice, mais, en s'exacerbant, elle favorise la dégénérescence des neurones.

\section{Hypothèse de travail : responsabilité de la dysbiose dans les déficits moteurs de la maladie de Parkinson}

L'existence, chez les patients parkinsoniens, d'une symptomatologie digestive, d'une dysbiose, et l'impact démontré du microbiote sur la fonction du système nerveux rendaient plausible une responsabilité du microbiote dans la survenue des déficits moteurs observés dans la maladie de Parkinson. Dans un article publié dans Cell fin 2016 [9], l'équipe de S.K. Mazmanian (Caltech, Californie, États-Unis) cherche à démontrer cette hypothèse et à identifier les composants du MI responsables de l'apparition de

\footnotetext{
${ }^{5}$ Cellule microgliale: cellule immunitaire de type macrophage résidente du SNC.
} 
ces déficits moteurs. En effet, l'hypothèse (controversée) d'une diffusion de type prion d'agrégats d' $\alpha$ Syn depuis le système nerveux entérique jusqu'au SNC via le nerf vague a été formulée.

\section{Le modèle murin utilisé pour l'étude}

S.K.Mazmanian et al. [9] utilisent un modèle murin appelé Thyl- $\alpha S_{y} n^{6}$ dit ASO (alpha-synuclein overexpressing) qui permet la surexpression spécifique de l'aSyn par les neurones. II s'agit du meilleur modèle actuel pour les formes sporadiques de la maladie de Parkinson. Chez ces souris, l'agrégation de l' $\alpha$ Syn entraîne des troubles moteurs et gastrointestinaux similaires à ceux qui sont observés chez les patients parkinsoniens [10]. Les déficits moteurs sont évalués à l'aide d'une batterie de tests de motricité, plus ou moins spécifiques en termes de muscles ciblés ${ }^{7}$. La diversité microbienne a, quant à elle, été mesurée par la méthode UniFrac ${ }^{8}$.

\section{Dysbiose et surexpression d' $\alpha$ Syn, deux éléments déterminants}

L'équipe de Mazmanian a mesuré l'impact du microbiote sur les déficits moteurs en utilisant des souris dites germ-free $^{9}$ (GF), dépourvues de MI, et des souris specific pathogens-free 10 (SPF), dont le microbiote mime une dysbiose. Ces deux phénotypes ont été établis chez des souris sauvages (WT) et des souris ASO surexprimant l' $\alpha$ Syn, ce qui permet d'observer le lien entre microbiote et $\alpha$ Syn. L'étude de la motricité des différentes souris montre l'absence d'anomalies chez les souris WT-GF et WT-SPF, alors que des déficits moteurs

\footnotetext{
${ }^{6}$ Thyl-aSyn : le promoteur Thyl, exprimé de manière spécifique dans les neurones, permet une surexpression spécifique de l'aSyn à la membrane des neurones.

${ }^{7}$ Les tests de motricité effectués concernent la mesure du temps de traversée de barres verticales et horizontales et l'étude de réflexes de membres spécifiques notamment les pattes arrières des souris dans les mouvements de fuite.

${ }^{8}$ Unifrac: cette méthode permet, par le séquençage des ARNr $16 S$ des populations microbiennes, l'analyse quantitative et qualitative d'un microbiote.

${ }^{9}$ Germ-free: qualifie des souris ne possédant pas de MI (axéniques).

${ }^{10}$ Specific pathogen-free : qualifie des souris dont le MI est exempt de souches connues comme étant pathogènes.
}

existent chez les souris ASO-SPF, mais pas chez les souris ASO-GF. Ainsi, chez ces souris ASO-SPF, les temps d'exécution des tâches motrices sont plus longs, et une raideur accentuée des membres inférieurs empêche la bonne réalisation des mouvements. Ces altérations sont absentes chez les souris ASO-GF, dont les caractéristiques motrices sont identiques à celles des souris WT. Ainsi, une dysbiose du MI, seule, ne serait pas suffisante pour induire des troubles moteurs; mais, lorsqu'elle est couplée à une surexpression d' $\alpha$ Syn, elle semble accentuer les déficits moteurs. Pour explorer ce lien entre composition du MI et $\alpha$ Syn, les chercheurs ont analysé par immunohistochimie, sur des coupes de cerveaux, les taux de protéines aSyn solubles (phosphorylées) et insolubles présentes sous forme d'agrégats dans les neurones, afin de déterminer si le MI influençait l'agrégation d'aSyn. On constate effectivement une proportion d'agrégats insolubles plus importante chez les souris ASO-SPF que chez les souris ASO-GF. Comme la maladie de Parkinson s'accompagne d'une importante neuro-inflammation, les chercheurs ont mesuré dans le modèle murin l'activité des cellules microgliales en réponse à la surexpression d' $\alpha$ Syn. Une reconstruction 3D, obtenue à partir des images d'immunomarquage avec un marqueur spécifique des cellules microgliales (Iba-1), a permis d'observer leur morphologie. Lorsqu'ils sont activés, ces macrophages réduisent la longueur et le nombre de leurs filopodes utilisés pour la détection d'agents pathogènes ou de sites d'inflammation en cas d'infection. Le volume de leur corps cellulaire augmente, conséquence d'une augmentation du métabolisme intracellulaire. Leur activation induit également une sécrétion des cytokines pro-inflammatoires TNF- $\alpha$ (tumor necrosis factor) et IL(interleukine)-6. De telles cellules microgliales activées étaient présentes dans le striatum des souris ASO-SPF. Fait intéressant, cette neuro-inflammation semble induite par le MI, car elle n'est pas retrouvée chez les souris ASO-GF (sans $M I$ ), et ce de manière conjointe à la surexpression d' $\alpha$ Syn, car elle n'est pas observée non plus dans les souris WT-SPF (sans $\alpha$ Syn, mais avec $M I$ ). En résumé, la neuro-inflammation et les déficits moteurs observés dans le modèle murin de la maladie de Parkinson sont modulés de manière concomitante par le $\mathrm{Ml}$ et la surexpression d'aSyn.

\section{Un coupable identifié : les acides gras à chaîne courte}

Les chercheurs se sont penchés sur les mécanismes à l'origine des troubles observés, et, dans un premier temps, ils ont testé la réversibilité de ces troubles, afin d'écarter leur origine développementale. Des souris SPF ont été rendues germ-free par un traitement antibiotique en période postnatale: souris $A B X$. À l'inverse, des souris GF ont été transplantées avec le MI de souris SPF: souris $\varepsilon X-G F$. Ces traitements ont été réalisés chez des souris WT et ASO afin d'étudier une potentielle modulation de l'aSyn sur les déficits moteurs. Les tests moteurs et les expériences de marquage de la microglie pratiqués chez les souris des groupes $A B X$ et $G F$ et celles des groupes EX-GF et SPF ont donné des résultats identiques aux phénotypes ASO et WT respectivement. Par conséquent, les voies de signalisation modulées par le $M I$ concernent des évènements acquis et réversibles, et donc indépendants du développement. Les chercheurs ont ensuite identifié les acides gras à chaîne courte $^{11}$ (AGCC) comme les responsables de l'induction des troubles associés au $M I$, en combinaison avec la surexpression d'aSyn. Ils ont observé une augmentation de la proportion d'AGCC dans les fèces des souris ASO-SPF comparées aux souris WT-GF et ASO-GF. Les chercheurs ont alors décidé de traiter des souris WT-GF et ASO-GF avec des AGCC pour essayer de mimer l'effet du MI. Le résultat fut concluant: le traitement

\footnotetext{
${ }^{11}$ Les AGCC sont les principaux produits issus de la fermentation bactérienne au niveau du côlon.
} 




Diffusion vers le SNC



Figure 1. La sécrétion d'AGCC et la formation conséquente d'agrégats d' $\alpha$-synucléine provoquent des troubles moteurs. Lors d'une perturbation du $\mathrm{MI}$ - ou dysbiose -, les bactéries se mettent à produire des acides gras à courtes chaînes carbonées (AGCC) qui, en diffusant dans le système nerveux central, provoquent une neuro-inflammation par la sécrétion de cytokines. Cette neuro-inflammation entraine une augmentation de la formation d'agrégats d' $\alpha$-synucléine qui provoquent la mort par apoptose de neurones dopaminergiques de la substance noire. De plus, la formation d'agrégats d' $\alpha$-synucléine favorise la dysbiose du microbiote intestinal, créant ainsi une signalisation à double sens qui accentue le phénomène. In fine, la perte de ces neurones régulant la réponse motrice via les synapses neuromusculaire résulte en l'apparition de troubles moteurs de type parkinsonien. AGCC : acides gras à courtes chaînes. IL-6 : interleukine 6 ; TNF $\alpha$ : tumor necrosis factor.

par AGCC induit, chez les souris ASOGF mais pas chez les souris WT-GF, des troubles moteurs et inflammatoires similaires à ceux qui sont observés chez les souris ASO-SPF et ASO-EX-GF.

Un cercle vicieux entre déséquilibre $d u$ $M I$ et agrégation d' $\alpha$ Syn participe aux dysfonctionnements moteurs dans la maladie de Parkinson

Après avoir identifié l'agent responsable des déficits moteur dans le MI, les chercheurs se sont alors intéressés au rôle de ces AGCC dans la dysbiose à l'origine des troubles moteurs, ainsi que dans l'agrégation d'aSyn. Pour cela, les chercheurs ont transplanté le MI de patients parkinsoniens et d'individus sains chez des souris ASO-GF et WT-GF. Puis, la qualité et la quantité en bactéries du MI ont été évaluées par UniFrac. On observe une conservation des caractéristiques du MI de l'individu donneur chez la souris hôte. D'autre part, on sait que si la diversité des MI est importante chez les individus sains, elle est, en revanche, très faible dans la population des patients parkinsoniens, révélant ainsi une signature microbienne corrélée à la maladie. Cependant, il s'établit une diversité en termes de populations bactériennes chez les souris hôtes, WT et ASO transplantées avec un même MI issu d'individus sains: l'agrégation de I'aSyn serait donc impliquée dans la création d'une dysbiose microbienne et dans la limitation de la diversité microbienne chez les patients. Réciproquement, on observe aussi une augmentation de l'agrégation de l' $\alpha$ Syn chez les souris WT-GF transplantées avec le MI issu d'un patient parkinsonien : la dysbiose promeut en retour l'agrégation de l'aSyn, et donc possiblement le développement de la maladie de Parkinson. Pour renforcer ce lien entre dysbiose et maladie de Parkinson, des tests moteurs ont été réalisés sur les souris WT et ASO 
transplantées avec des MI de patients parkinsoniens ou d'individus sains. Ils ont mis en évidence une aggravation des déficits moteurs chez les souris ASO par rapport aux souris WT, mais aussi par rapport aux souris ASO transplantées avec un MI d'individus sains. Ainsi, le déséquilibre du MI des patients parkinsoniens favorise l'agrégation d'aSyn et, réciproquement, les deux effets concourant à l'installation de déficits moteurs.

\section{Conclusions et perspectives}

Ces résultats démontrent le rôle du MI dans l'apparition des troubles moteurs et la neuro-inflammation de la maladie de Parkinson, et identifient les AGCC comme un des composants directement responsables. Le MI favorise l'agrégation d'aSyn qui, à son tour, exacerbe la dysbiose, constituant ainsi un cercle vicieux. Cependant, des questions restent ouvertes sur la diffusion des AGCC dans le SNC et la formation d'agrégats d'aSyn. L'étude ne permet pas d'infirmer ou de confirmer l'hypothèse de Braak [11] concernant la diffusion des agrégats d'aSyn depuis l'intestin jusqu'au SNC, car on ne sait pas si la signalisation induite par les AGCC entraîne la formation des agrégats d' $\alpha$ Syn au niveau du système nerveux entérique ou du SNC. Cependant, la neuro-inflammation observée au niveau du SNC est modulée par les AGCC, ce qui suggère une fonction régulatrice de ces composés au niveau du cerveau à moins que cette signalisation utilise des intermédiaires effectuant le relai entre l'intestin et le SNC. De même, on ne sait pas en retour comment l'agrégation d'aSyn favorise la dysbiose. Alors qu'elle entraîne la dégénérescence spécifique des neurones dopaminergiques dans la maladie de Parkinson, l'article montre que l'aSyn entraîne une dégénérescence d'espèces bactériennes spécifiques expliquant l'homogénéité des microbiotes observés chez les patients parkinsoniens. Ainsi, il serait intéressant, dans la perspective de potentielles thérapies, d'étudier le mécanisme de dégénérescence cellulaire provoqué par l'action spécifique de l' $\alpha$ Syn.

$\varepsilon n$ somme, bien que les chercheurs aient identifié l'interaction entre les deux maillons que sont l' $\alpha$ Syn et la dysbiose dans la maladie de Parkinson, nous ne connaissons pas l'élément responsable de cette signalisation réciproque ni même l'origine du dysfonctionnement: qui de l'œuf ou de la poule entre l'agrégation d'aSyn et la dysbiose? Sur un plan thérapeutique, l'observation d'une réversibilité des déficits moteurs induits par la dysbiose permet d'envisager de rétablir un microbiote sain chez les patients parkinsoniens. Une des stratégies pourrait être la transplantation de microbiote [12] $(\rightarrow)$. Autre piste, la neuroinflammation exacer-

$(\rightarrow)$ Voir la Synthèse de J.C. Lagier et

D. Raoult, $m / s n^{\circ} 11$, novembre 2016, page 991

bée qui favorise l'agrégation de l'aSyn. Un traitement anti-inflammatoire associé à une restauration du microbiote intestinal pourrait permettre d'améliorer les déficits moteurs et la qualité de vie des patients parkinsoniens. $\diamond$

When germs get involved in Parkinson's disease

\section{LIENS D'INTÉRÊT}

Les auteurs déclarent n'avoir aucun lien d'intérêt concernant les données publiées dans cet article.

\section{RÉFÉRENCES}

1. Ley RE, Peterson DA, Gordon JI. Ecological and evolutionary forces shaping microbial diversity in the human intestine. Cell 2006 ; $124: 837-48$.

2. Selkrig J, Wong P, Zhang X, Pettersson S. Metabolic tinkering by the gut microbiome: Implications for brain development and function. Gut Microbes 2014 ; $5: 369-80$.

3. Yano JM, Yu K, Donaldson GP, et al. Indigenous bacteria from the gut microbiota regulate host serotonin biosynthesis. Cell 2015 ; 161 : 264-76.

4. Diaz Heijtz R, Wang $S$, Anuar F, et al. Normal gut microbiota modulates brain development and behavior. Proc Natl Acad Sci USA 2011 ; 108 : 3047-52

5. Möhle L, Mattei D, Heimesaat MM, et al. Ly6C (hi) monocytes provide a link between antibiotic-induced changes in gut microbiota and adult hippocampal neurogenesis. Cell Rep 2016 ; 15 : 1945-56.

6. Mayer $E A$, Padua D, Tillisch K. Altered brain-gut axis in autism: comorbidity or causative mechanisms? BioEssays 2014 ; 36 : 933-9.

7. Schroeder BO, Bäckhed F. Signals from the gut microbiota to distant organs in physiology and disease. Nat Med 2016; 22 : 1079-89.

8. Scheperjans F, Aho V, Pereira PA, et al. Gut microbiota are related to Parkinson's disease and clinical phenotype. Mov Disord $2015 ; 30: 350-8$.

9. Sampson TR, Debelius JW, Thron T, et al. Gut microbiota regulate motor deficits and neuroinflammation in a model of Parkinson's disease. Cell $2016 ; 167: 1469-80$

10. Chesselet MF, Richter F, Zhu C, et al. A progressive mouse model of Parkinson's disease: the Thyl-aSyn (Line 61) mice. Neurotherapeutics 2012 ; 9 : 297-314.

11. Del Tredici K, Braak H. A not entirely benign procedure: progression of Parkinson's disease. Acta Neuropathol $2008 ; 115: 379-84$.

12. Lagier JC, Raoult. Greffe de microbiote fécal et infections. Med Sci (Paris) 2016 ; 32 : 991-7.

\section{LA FONDATION PREMUP : UN OPÉRATEUR DE TERRAIN EN PÉRINATALITÉ RECONNU POUR SON EXCELLENCE ET SON INTERDISCIPLINARITÉ}

La Fondation de coopération scientifique PremUp, unique en Europe, intervient sur la prévention du handicap à la naissance, par la protection de la santé de la femme enceinte et du nouveau-né.



ONDATION DE COOPEERAIION SCIENTIFIQUÉ
SUR LA GROSSESSE ET LA PRÉMATURITÉ

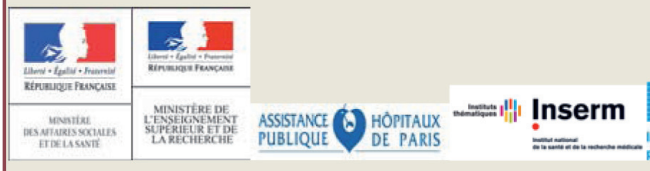

